\title{
Crecimiento del Cangrejo de Tierra Cardisoma Guanhumi en Puerto Rico
}

\section{Growth of the Land Crab Cardisoma Guanhumi in Puerto Rico}

INFORMACIÓN DEL

\section{ARTÍCULO}

Fecha de recepción: 27 de Mayo de 2020.

Fecha de aceptación: 27 de julio de 2020
${ }^{1}$ Departamento de Biología, Universidad de Puerto Rico.

E-mail: concepcion.rodriguez@upr.edu Código ORCID:

https://orcid.org/0000-0001-7622-7381

CITACIÓN: Rodríguez-Fourquet, C. (2020). Crecimiento del Cangrejo de Tierra Cardisoma Guanhumi en Puerto Rico. Investigatio, 14, 38-45. doi:10.31095/investigatio.2020.14.4

\section{ENLACE DOI:}

http://dx.doi.org/10.31095/investigatio. 2020.14.4

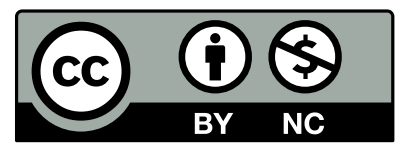

Concepción Rodríguez-Fourquet ${ }^{1}$

\section{Resumen}

Para los cangrejos terrestres, el proceso de liberación del integumento en el proceso de muda representa un momento de vulnerabilidad debido al riesgo de desecación y la disponibilidad de agua para el proceso de muda. El objetivo de este estudio es determinar el incremento en crecimiento del cangrejo de tierra Cardisoma guanhumi. Se realizó un estudio de marca-recaptura para estimar el crecimiento de los cangrejos. El estudio se realizó en seis localidades en Puerto Rico. Encontramos que el incremento en crecimiento es de $8.7 \%$ y que hay una diferencia entre los lugares de estudio. Este incremento es mayor que lo reportado anteriormente. La diferencia entre los lugares de estudio se debe a diferencias en la estructura de los bosques. Los bosques con mayor cobertura vegetal y menos densidad de borde favorecen un incremento en crecimiento. La conservación del hábitat del cangrejo es esencial para la sobrevivencia de esta especie.

\section{Palabras Clave:}

Cangrejo de tierra; Cardisoma guanhumi; crecimiento; ecdisis; muda; Puerto Rico.

\section{Clasificación JEL: Q0.}

\section{Abstract}

The land crabs' integument release process during molting represents a moment of vulnerability due to the desiccation risk and the lack of water availability for the molting. The aim of this study is to ascertain Cardisoma guanhumi growth increase. A marking-recapture study was conducted to estimate crab growth. The study was carried out in six Puerto Rico locations. It was found that growth increment is $8.7 \%$, which is larger than previously reported; this increment varies among study sites. Forests with higher vegetation coverage and lower edge density favor a larger growth increment. Crab's habitat conservation is essential for the species' survival.

\section{Keywords:}

Cardisoma guanhumi, ecdysis, growth, land crab, molting, Puerto Rico.

JEL Classification: Q0. 


\section{Introducción}

A medida que los organismos se mueven a través de sus ciclos de vida estos nacen, crecen, maduran, se reproducen y mueren. En todos estos procesos, el crecimiento es un factor vital para completar el ciclo de vida. En los crustáceos el crecimiento se lleva a cabo por la liberación del integumento, proceso llamado ecdisis, y la producción de un integumento nuevo. A ambos procesos se le llama muda (Skinner, 1985). Para los cangrejos terrestres este proceso representa un momento de vulnerabilidad debido al riesgo de desecación y de poca disponibilidad de agua (Hartnoll, 1988). Algunos cangrejos terrestres llevan a cabo la muda enterrados en cuevas (Henning, 1975) y de esta forma minimizan estos riesgos. E1 agua necesaria para la muda, la obtienen de diferentes formas ya que algunos cangrejos tienen agua en sus cuevas y otros acumulan agua en sus tejidos o en la cavidad pericardial (Bliss, 1963). La tasa de crecimiento de los cangrejos terrestres depende de dos factores: el incremento en tamaño y el tiempo entre mudas. Ambos factores pueden variar dependiendo de la etapa del ciclo de vida y se espera a medida que aumente la edad del cangrejo el incremento en tamaño disminuya $y$ el tiempo entre mudas aumente (Hartnoll, 1988).

Con respecto a la distribución y ecología de Cardisoma guanhumi, este habita las áreas costeras en la costa este tropical y subtropical de América, el Caribe y las Bermudas (Chace \& Hobbs, 1969). Cardisoma guanhumi vive en cuevas en suelos fangosos cerca de manglares, pantanos y arroyos donde las cuevas pueden alcanzar las aguas subterráneas (Feliciano, 1962; Herreid \& Gifford, 1963). Los cangrejos se alimentan de hojas caídas al suelo de las diferentes especies de mangles, de hierbas, frutos, semillas y flores. El crecimiento de esta especie ha sido asociado a la cantidad de hojarasca en el suelo. A mayor cantidad de hojarasca, mayor el tamaño de los cangrejos (Rodríguez-Fourquet \& Sabat, 2009).

Esta es una especie de importancia comercial a pequeña escala en Puerto Rico ya que se ha capturado comercialmente desde principios de la década de 1930. Desde ese momento la demanda por el cangrejo se ha mantenido a pesar de ser una especie protegida por ley. Hoy día los datos recopilados de los monitoreos de las poblaciones sugieren que algunas de estas, están sobre explotadas y que se necesita hacer cumplir la reglamentación que protege a estos cangrejos.

Se conoce poco sobre el crecimiento de $C$. guanhumi en Puerto Rico por lo que el objetivo de este estudio es estimar el incremento en crecimiento de esta especie.

\section{Materiales y métodos}

Recopilamos datos mensuales sobre el ancho de cefalotórax (carapacho) y el sexo de los cangrejos en seis lugares de estudio en Puerto Rico. Los seis lugares de estudio fueron Refugio de Vida Silvestre de Boquerón (Boquerón), Cabo 
Rojo (18.017172, -67.156591); Estación Naval Roosevelt Roads (Comisaria), Ceiba (18.228379, -65.641509), Las Cabezas de San Juan (Redondel), Fajardo (18.380776, -65.626048), Punta Ballena (Ballena), Guánica (17.954553, -66.864514); Estación Naval Roosevelt Roads (Los Machos), Ceiba (18.261733, -65.629659); y Las Cabezas de San Juan (Canalejo), Fajardo (18.378247, -65.617589).

En cada uno de los lugares de estudio, elegimos al azar y marcamos una parcela de $100 \mathrm{~m} 2$. Dentro de esa parcela, colocamos trampas en todas las cuevas cuyo diámetro fuese de más de $4 \mathrm{~cm}$. Las trampas consistían en tubos de PVC (Figura 1) que se colocaron en la entrada de la cueva. Se utilizaron trampas de cuatro tamaños diferentes $(4,5,8,10 \mathrm{~cm}$ de diámetro) y no se utilizó cebo en las trampas. Las trampas se colocaron después de las 4:00 PM y se recuperaron antes de las 12:00 AM del día siguiente. El muestreo fue mensual durante 18 meses en Redondel y Comisaría; durante 17 meses en Boquerón, Los Machos y Canalejo; y durante 11 meses en Ballena.

A cada cangrejo capturado se le tomaron medidas de ancho del carapacho y se determinó el sexo. Los individuos con un ancho de carapacho de $50 \mathrm{~mm}$ o más se marcaron con un "PIT tag" (transpondedor integrado pasivo). El "PIT tag" es un dispositivo de

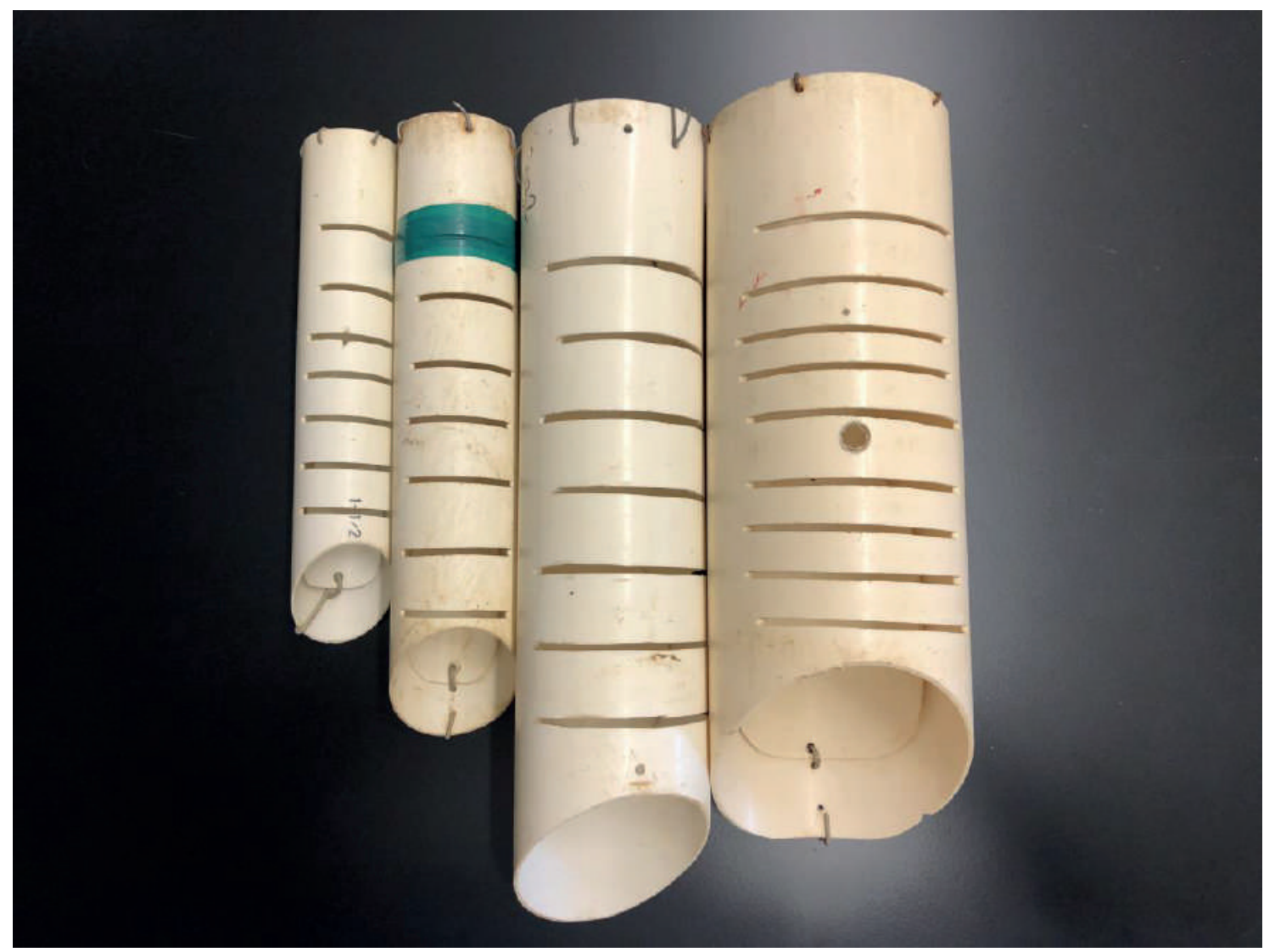

Figura 1. Trampas utilizadas para la captura de Cardisoma guanhumi. Se muestran los cuatro tamaños de trampas. Cada trampa tiene una tapa que permite la entrada del cangrejo, pero no la salida de este.

40

INVESTIGATIO No. 14, Edición Especial, septiembre 2020, pp. 38-45,

(C) Universidad Espíritu Santo - UEES

ISSN: 1390 - 6399 - ISSN-e: 2602 - 8336 
radiofrecuencia que transmite un código alfanumérico único a un lector (AVID Power Tracker II). Los "PIT tags" son de vidrio $y$ tienen forma cilíndrica. Utilizamos dos tamaños, AVID (C) $12 \mathrm{~mm}$ tag y Biomark $11.5 \mathrm{~mm}$ con peso promedio de 0.06 gramos. El "PIT tag" se implantó con una jeringa entre la $4^{\mathrm{a}} \mathrm{y}$ la $5^{\text {a }}$ pata de caminar en el lado derecho del cangrejo. Después de que se realizaron las mediciones y las marcas, todos los animales capturados fueron liberados inmediatamente a la cueva donde fueron capturados.

El crecimiento se determinó a partir de los cangrejos recapturados que llevaron a cabo el proceso de muda durante el período de estudio. Las diferencias en el ancho del cefalotórax entre las temporadas de 2001 y 2002 se consideraron crecimiento. Si el cangrejo fue recapturado dos veces o más durante la misma temporada, tomamos un promedio de las medidas de ancho de cefalotórax dentro de esa temporada, lo consideramos como medida inicial si fue en el 2001 y como medida final si fue en el 2002. Se observó la heteroquelia y se anotó la pérdida de apéndices debido al manejo de los animales.

Para comparar el crecimiento entre machos y hembras y entre los sitios, se utilizó un análisis de varianza (ANOVA) de un factor. Se realizó una regresión para determinar la relación entre el tamaño inicial y el crecimiento. El número observado y esperado de cangrejos que mudaron por sitio se comparó con una prueba de $\chi 2$.

\section{Resultados}

Ochenta y cinco cangrejos (85) dentro de los cuales hay treinta y nueve (39) hembras y cuarenta y seis (46) machos mudaron y crecieron durante el período de estudio en los seis lugares estudiados (Tabla 1). La mayor cantidad de cangrejos que mudaron se capturaron en Redondel. Siete de los cangrejos mudaron dos veces durante el período de estudio. Un cangrejo en Comisaría creció $14.1 \mathrm{~mm}$ entre mayo de 2001 y mayo de 2002. Los cangrejos que perdieron las quelas, las recuperaron después de la muda y se mantuvo la heteroquelia en el

\section{Tabla 1}

Número de recapturas e individuos de Cardisoma guanhumi que mudaron entre mayo 2001 y noviembre de 2002 en seis lugares de estudio en Puerto Rico

\begin{tabular}{cccccccc}
\hline \multirow{2}{*}{$\begin{array}{c}\text { Lugar } \\
\text { Número de }\end{array}$} & Mudas & \multicolumn{2}{c}{ Mudas } & & $\begin{array}{c}\text { Mudados } \\
\text { veces } \\
\text { recapturas }\end{array}$ & hembras & $\begin{array}{c}\text { Muda dos } \\
\text { veces } \\
\text { machos }\end{array}$ \\
\hline Boquerón & 25 & 6 & 4 & 2 & 0 & 0 \\
Comisaría & 26 & 9 & 4 & 6 & 0 & 0 \\
Redondel & 96 & 54 & 26 & 28 & 4 & 3 \\
Ballena & 11 & 4 & 2 & 2 & 0 & 0 \\
Los Machos & 13 & 6 & 1 & 5 & 0 & 0 \\
Canalejo & 23 & 5 & 2 & 3 & 0 & 0 \\
Total & 194 & 85 & 39 & 46 & 4 & 3 \\
\hline
\end{tabular}

Fuente: Elaboración propia. 
lado original, no cambiaron de posición. No hubo diferencias entre el número de cangrejos que mudó con respecto a los sitios de estudios $(\chi 2=0.000, \mathrm{df}=1, \mathrm{p}=$ 0.983).

El incremento de tamaño promedio fue $5.74 \mathrm{~mm}( \pm 2.3 \mathrm{~mm}$ desviación estándar). Este incremento en tamaño representa $8.7 \%$ de crecimiento. En las hembras, el promedio de incremento en tamaño fue $5.6 \mathrm{~mm}( \pm 2.6 \mathrm{~mm}$ desviación estándar) mientras que para los machos fue de $5.8 \mathrm{~mm}( \pm 2.3 \mathrm{~mm}$ desviación estándar). No hubo diferencia significativa en el incremento en tamaño entre las hembras y los machos ( $\mathrm{t}=-0.64$; $\mathrm{df}=81 ; \mathrm{p}=0.521) . \quad$ Sin embargo, encontramos una diferencia significativa entre los lugares de estudio al combinar los datos de hembras y machos, pero no encontramos una diferencia significativa en la interacción entre lugar de estudio y sexo (Tabla 2). El mayor incremento en crecimiento promedio se observó en Comisaría (7. $7 \mathrm{~mm}$ y el más bajo en Ballena (Figura 2). No encontramos una relación entre el tamaño de los cangrejos (ancho del cefalotórax) y el incremento en crecimiento.

\section{Tabla 2.}

Análisis de varianza para el incremento en crecimiento de Cardisoma guanhumi para seis lugares de estudio y por sexo en Puerto Rico. GL = grados de libertad; SMA = suma de cuadrados ajustada; MCA = media cuadrática ajustada

\begin{tabular}{llllll}
\hline Fuente & GL & SMA & MCA & Valor F & Valor P \\
\hline Lugar & 5 & 65.481 & 13.096 & 2.82 & 0.022 \\
Sexo & 1 & 2.296 & 2.296 & 0.49 & 0.484 \\
Lugar*Sexo & 5 & 38.678 & 7.736 & 1.67 & 0.153 \\
Error & 73 & 338.689 & 4.640 & & \\
Total & 84 & 444.214 & & & \\
\hline
\end{tabular}

Fuente: Elaboración propia.

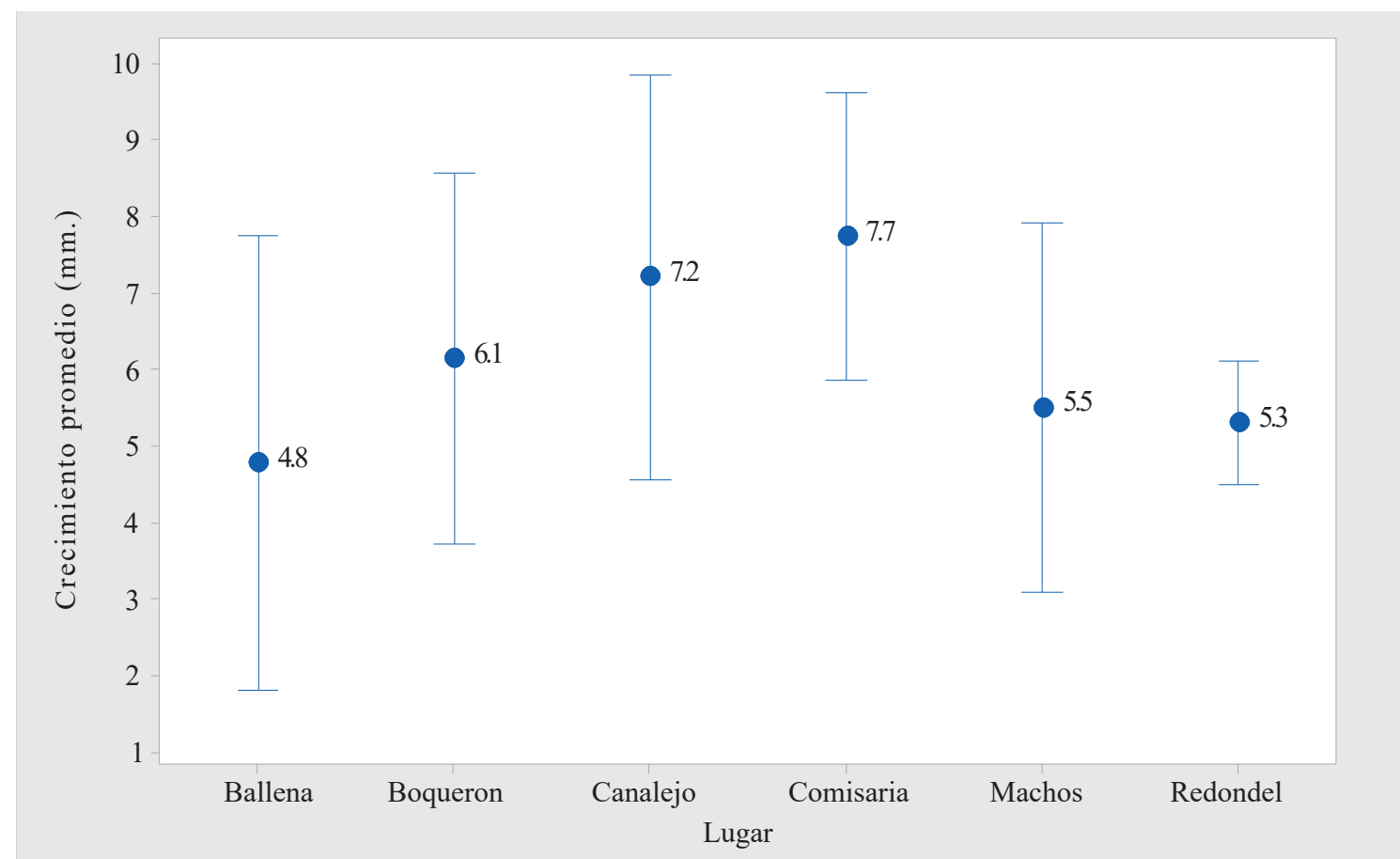

Figura 2. Crecimiento promedio de Cardisoma guanhumi (hembras y machos) en seis lugares de estudio en Puerto Rico. Las barras de error representan el intervalo de confianza del promedio al $95 \%$.

Fuente: Elaboración propia. 


\section{Discusión}

Este estudio presenta el crecimiento de Cardisoma guanhumi en Puerto Rico basado en las recapturas en seis lugares de estudio. Las recapturas y la frecuencia de muda variaron entre los lugares de estudio. Sin embargo, podemos inferir que la probabilidad de muda es similar en todas las áreas de estudio, según la prueba de independencia. Esto nos indica que todos los lugares de estudio son hábitats favorables para la presencia de C. guanhumi.

El promedio del incremento en tamaño para los cangrejos de Puerto Rico se establece en un $8.7 \%$. Este incremento es mayor que el incremento de $6 \%$ reportado por Feliciano (1962) para $C$. guanhumi en Puerto Rico. Henning (1975) estable que el incremento en crecimiento es de cerca de $10 \%$ para los cangrejos juveniles (menores de $10 \mathrm{~mm}$ ancho de cefalotórax) y menos de 5\% para los cangrejos mayores de $50 \mathrm{~mm}$ de ancho de cefalotórax. En el presente estudio todos los cangrejos eran mayores de $50 \mathrm{~mm}$ de ancho de cefalotórax. El incremento en crecimiento reportado en este estudio, también es mayor al reportado para Gecarcoidea natalis, el cangrejo rojo de la Isla Navidad perteneciente a la misma familia Gecarcinidae a la que pertenece $C$. guanhumi, cuyo incremento en tamaño fue de $2.9 \%$ (Green, 2004).

Las diferencias en incremento en tamaño entre los estudios de C. guanhumi pueden ser debido a las condiciones en las cuales se encontraban los cangrejos.
En el caso de Henning (1975), este reporta un incremento mayor al del presente estudio y reporta que las medidas de crecimiento se realizaron en el laboratorio. Es posible que los cangrejos no estuvieran expuestos a los procesos naturales como depredación, competencia, recibieron alimento y agua en abundancia permitiendo que el incremento en muda fuera mayor. Por otro lado, Feliciano (1962) reporta que los datos de incremento en tamaño se obtuvieron de 11 animales silvestres sin ofrecer más detalles al respecto y reporta un incremento en tamaño menor al del presente estudio. En este estudio la muda ocurrió bajo condiciones naturales de luz, temperatura, humedad y expuestos a todos los procesos naturales de competencia y depredación. Consideramos que estas son las causas en las diferencias en incremento en muda entre los diferentes estudios y no por causas naturales o cambios en el ecosistema.

El incremento en tamaño es diferente entre las diferentes áreas de estudio. Es mayor para Canalejo y Comisaría y menor en Ballena y Redondel. La estructura y composición de los bosques entre los lugares de estudio son diferentes en términos de cantidad de hojarasca, número de árboles, área basal, densidad de borde, nivel de disturbio y composición de especies (RodriguezFourquet \& Sabat, 2009). Pensamos que la estructura y edad del bosque juegan un papel importante en el crecimiento de los cangrejos, ya que estos proveen diferentes tipos y calidad de alimentos. Canalejo y Comisaría son bosques que proveen mejores condiciones para el 
crecimiento de los cangrejos debido a una mayor cobertura vegetal, poca densidad de borde y pocos disturbios (Rodríguez Fourquet, 2004 y Rodríguez-Fourquet \& Sabat, 2009). Por otro lado, Ballena y Redondel son áreas con menor cobertura vegetal y alta abundancia de cangrejos indicativo de posible competencia por alimento (Rodríguez-Fourquet, 2004; Rodríguez-Fourquet \& Sabat, 2009) que a su vez se puede reflejar en un incremento en crecimiento menor debido a la poca cantidad y calidad de alimentos.

Esperábamos encontrar una
disminución del incremento en crecimiento según aumenta el ancho del cefalotórax, debido a que los cangrejos más viejos crecen menos (Henning, 1975). Sin embargo, no observamos esa relación. Pensamos que la relación no se observó porque solo medimos el crecimiento en cangrejos adultos $(>50$ $\mathrm{mm}$ de ancho de carapacho) cuyo crecimiento podría ser constante durante la edad adulta. Para poder comprobar ese efecto es necesario tomar muestras de todas las tallas en la población asumiendo que los cangrejos juveniles tienen incrementos en tamaños mayores hasta llegar a la etapa de madurez sexual o adultez.

Existe poca literatura sobre el crecimiento de Cardisoma guanhumi y este estudio provee información nueva sobre las poblaciones de esta especie para Puerto Rico. El incremento en crecimiento en las poblaciones de $C$. guanhumi en Puerto Rico es mayor que los valores reportados en la literatura y varía según la localidad debido a la estructura y composición de los bosques. El estimar el crecimiento de C. guanhumi es relevante para el manejo y la conservación de la especie. Al conocer sobre el incremento en tamaño y las condiciones del hábitat del organismo, podemos establecer medidas para la conservación de hábitat con las características favorables para crecimiento. Por ejemplo, se puede recomendar la designación de áreas costeras con poco disturbio, con alta diversidad de plantas y gran cantidad de hojarasca en el suelo como áreas críticas para el crecimiento de C. guanhumi.

\section{Agradecimientos}

Este estudio recibió el apoyo financiero de Programa de Colegio Sea Grant Universidad de Puerto Rico en Mayagüez, Seed Money Program, PD-225-R/L.b.-31 y del programa AGEPNSF. Agradezco al Departamento de Recursos Naturales y Ambientales de Puerto Rico, Fideicomiso de Conservación de Puerto Rico. Un agradecimiento particular a Miguel Figuerola y a todos los estudiantes que ayudaron con el trabajo de campo.

\section{Referencias}

Bliss, D. E. (1963). The pericardial sacs of terrestrial Brachyura. In T. H. Waterman (Ed.), Phylogeny and evolution of Crustacea (First, pp. 59-78). New York: Academic Press.

Chace, F., \& Hobbs, H. (1969). The freshwater and terrestrial decapod crustaceans of the West Indies with special reference to Dominica. United States National Museum Bulletin 292. 
Feliciano, C. (1962). Notes on the Ecological Importance of the Land Crab Cardisoma gunhumi, Latreille of Puerto Rico. Mayaguez.

Green, P. T. (2004). Field observations of moulting and moult increment in the red land crab, Gecarcoedea natalis (Brachyura, Gecarcinidae), on Christmas Island (Indian Ocean). Crustaceana, 77(1), 125-128. doi.org/10.1163/156854004323 037937

Hartnoll, R. G. (1988). Growth and Molting. In W. W. Burggren \& McMahon Brian R. (Eds.), Biology of Land Crabs (First, p. 479). New York: Cambridge University Press.

Henning, H. H. (1975). Ökethologische, ethologische und sinnesphysiologische Untersuchungen an der Landkrabbe Cardisoma guanhumi Latreille (Decapoda, Brachyura). Forma et Functio, 8, 253-304.

Herreid, C. F., \& Gifford, C. A. (1963). The Burrow Habitat of the Land Crab, Cardisoma guanhumi (Latreille). Ecology, 44(4), 773-775. doi.org/10.2307/1933027

Rodriguez-Fourquet, C. (2004). Abundance and demography of the land crab Cardisoma guanhumi in Puerto Rico. University of Puerto Rico.

Rodríguez-Fourquet, C., \& Sabat, A. M. (2009). Effect of harvesting, vegetation structure and composition on the abundance and demography of the land crab Cardisoma guanhumi in Puerto Rico. Wetlands Ecology and Management, 17(6). doi.org/10.1007/s11273-009-9139-5

Skinner, D. M. (1985). Molting and regeneration. The biology of Crustacea, 9, 43-146. 